\title{
ON THE BRENNAN CONJECTURE
}

\author{
ENRIQUE VILlamor AND FENG XiaO
}

\begin{abstract}
In this paper we study the conformal mappings of some symmetric simply connected domains in the complex plane whose boundary are fractal trees. In particular we present, based on the alternative simplified approach of Carleson and Makarov to the Brennan conjecture, see $[\mathrm{CM}]$, some evidence towards the truth of this conjecture.
\end{abstract}

\section{Introduction}

Let $\Omega$ be a simply connected domain with at least two boundary points in the extended complex plane, and let $\Phi$ be a conformal mapping of $\Omega$ onto the open unit disk. Is

$$
\iint_{\Omega}\left|\Phi^{\prime}\right|^{p} d x d y<\infty
$$

for $4 / 3<p<4$ ?

If $p=2$ this integral represents the area of the unit disk and therefore is finite. If $\Omega$ is the plane slit along the negative real axis, then the integral converges for $4 / 3<p<4$ and diverges for $p=4 / 3$ and $p=4$. This follows easily from a direct computation.

In $[\mathrm{Br}]$, Brennan proved that there exists a constant $\tau>0$ which does not depend on $\Omega$ such that

$$
\iint_{\Omega}\left|\Phi^{\prime}\right|^{p} d x d y<\infty
$$

for $4 / 3<p<3+\tau$.

He also proved that for a large class of domains, including starlike and close to convex domains, $p=4$ is the correct upper bound.

In $[\mathrm{Po}$, Pommerenke proved that

$$
\iint_{\Omega}\left|\Phi^{\prime}\right|^{p} d x d y<\infty
$$

1991 Mathematics Subject Classification. Primary 30C20.

Key words and phrases. Extremal length, conformal mapping and Green's function.

Received June 24, 1996; revised August 12, 1997. 
for $4 / 3<p<3.399$, which gives the best known lower bound for $\tau$.

Recently Carleson and Makarov [CM] have reformulated Brennan's conjecture as follows.

Let $D$ be a simply connected domain and $\infty, a_{1}, \ldots, a_{m} \in \partial D$. Let $\Phi$ be a conformal mapping of the upper half plane onto $D$ satisfying $\Phi(z) \sim z^{2}$ as $z \rightarrow \infty$. Suppose the points $x_{j} \in R$ are such that $\Phi\left(x_{j}\right)=a_{\jmath}, \Phi^{\prime}\left(x_{j}\right)=0$ and $\Phi^{\prime \prime}\left(x_{\jmath}\right)$ exists for $j=1, \ldots, m$.

Then define $\beta$, by

$$
\beta_{\jmath}=\beta\left(D, a_{j}\right)=\frac{2}{\left|\Phi^{\prime \prime}\left(x_{j}\right)\right|}, \quad \jmath=1, \ldots, m .
$$

There are several equivalent definitions for these quantities. Carleson and Makarov proved that the Brennan's conjecture is equivalent to prove that for every configuration $\left(D, \infty, a_{1}, \ldots, a_{m}\right)$ as above,

$$
\sum_{j=1}^{m} \beta_{j}^{2}<1 \text {. }
$$

Using Schiffer's variation method, Carleson and Makarov proved that the above inequality holds for $m=2$.

They also showed in their paper that without loss of generality, we can consider $D$ to be a simply connected domain as in [V].

Namely, consider the domain $\Theta=C \backslash\left\{(-\infty, 0] \cup\left(\cup_{j=1}^{N}\left[0, a_{j}\right]\right)\right\}$, where $\left[0, a_{j}\right]$ is the line segment joining these two points. Let $a_{\jmath}=R_{j} e^{i \theta_{j}}$, where $-\pi<\theta_{1}<$ $\theta_{2}<\cdots<\theta_{N}<\pi$ and $0<R_{j}<\infty, N>2$. Consider the conformal mappings $F_{j}(z)$, $j=1, \ldots, N$ mapping $\Theta$ onto the domain $H=C \backslash(-\infty, 0]$, such that $F_{j}\left(a_{\jmath}\right)=0$, and $\lim _{z \rightarrow \infty}\left|F_{j}(z) / z\right|=1, j=1, \ldots, N$. Then

$$
\beta_{\jmath}=\lim _{z \rightarrow a_{j}}\left|\frac{F_{j}(z)}{z-a_{\jmath}}\right|=\left|F_{j}^{\prime}\left(a_{j}\right)\right| \quad \text { for } \quad \jmath=1, \ldots, N .
$$

We are going to study the $\lim \sup _{N \rightarrow \infty}\left(\sum_{j=1}^{N} \beta_{j} / \sqrt{N}\right)$, since if this limit is greater than 1 it follows by Schwarz's inequality that

$$
\begin{aligned}
\sum_{j=1}^{N} \frac{\beta_{\jmath}}{N} & =\sum_{j=1}^{N} \frac{\beta_{j}}{\sqrt{N}} \frac{1}{\sqrt{N}} \\
& \leqq \sqrt{\sum_{j=1}^{N} \frac{\beta_{j}^{2}}{N}} \sqrt{\sum_{j=1}^{N} \frac{1}{N}} \\
& =\sqrt{\sum_{j=1}^{N} \frac{\beta_{j}^{2}}{N}}
\end{aligned}
$$

and hence

$$
1<\sum_{j=1}^{N} \frac{\beta_{j}}{\sqrt{N}} \leqq \sqrt{\sum_{j=1}^{N} \beta_{j}^{2}}
$$

for $N$ large enough, and thus, by the Carleson-Makarov's criterion, the Brennan's conjecture is false. 
From now on, we shall denote by $C$ an absolute positive constant whose value can change from line to line, and use the notation $A \approx B$ to mean that $(1 / C) A \leqq B \leqq C A$ for some $C$.

Remark. Concerning the inequality $\sum_{j=1}^{N} \beta_{j}>\sqrt{ } \bar{N}$, for $N=2$ and $a_{1}=\bar{a}_{2}$, it appears that the largest $\beta_{1}=\beta_{2}$ occurs when $\arg a_{2} \approx .22 \approx \pi / 13$ and then $\beta_{1}=$ $\beta_{2} \approx 2 / 3$. Thus, $\beta_{1}+\beta_{2} \approx 1.33$, which is about six per cent less than $\sqrt{2}$.

\section{Preliminaries and related results}

Let $\Omega$ be a region in the complex plane, and $\Gamma$ a set whose elements $\gamma$ are rectifiable curves in $\Omega$. Then for a nonnegative Borel measurable function $\rho$, every $\gamma$ has a well-defined $\rho$ length

$$
L(\gamma, \rho)=\int_{\gamma} \rho|d z|,
$$

which may be infinite, and $\Omega$ has a $\rho$ area

$$
A(\Omega, \rho)=\iint_{\Omega} \rho^{2} d x d y .
$$

If we introduce the minimum length of the whole set $\Gamma$ as

$$
L(\Gamma, \rho)=\inf _{\gamma \in \Gamma} L(\gamma, \rho),
$$

then we can form the homogeneous expression

$$
\frac{L(\Gamma, \rho)^{2}}{A(\Omega, \rho)} \text {. }
$$

The set of all these ratios is conformally invariant, thus so is the least upper bound. Therefore, we define the extremal length of $\Gamma$ in $\Omega$ as

$$
\lambda_{\Omega}(\Gamma)=\sup _{\rho} \frac{L(\Gamma, \rho)^{2}}{A(\Omega, \rho)}
$$

where $\rho$ is subject to the condition $0<A(\Omega, \rho)<\infty$.

There are several alternative definitions for $\lambda_{\Omega}(\Gamma)$ obtained by using different normalizations. For instance, $\lambda_{\Omega}(\Gamma)$ is equal to $\sup _{\rho} L(\Gamma, \rho)^{2}$ when $\rho$ is subject to the condition $0<A(\Omega, \rho) \leqq 1$. Similarly, we say that $\rho$ is admissible if $L(\Gamma, \rho) \geqq 1$, and define the modulus of $\Gamma$ with respect to $\Omega$ as $M_{\Omega}(\Gamma)=$ $\inf _{\rho} A(\Omega, \rho)$ for admissible $\rho$, then $\lambda_{\Omega}(\Gamma)$ is the reciprocal of the modulus $M_{\Omega}(\Gamma)=\left(1 / \lambda_{\Omega}(\Gamma)\right)$.

The conformal invariance of both $M_{\Omega}(\Gamma)$ and $\lambda_{\Omega}(\Gamma)$ is an immediate consequence of the definition.

Let $\Omega$ be an open set and let $E_{1}, E_{2}$ be two disjoint sets in the closure of $\Omega$. Take $\Gamma$ to be the set of connected $\operatorname{arcs}$ in $\Omega$ which join $E_{1}$ and $E_{2}$. Then 
the extremal length $\lambda_{\Omega}(\Gamma)$ is called the extremal distance between $E_{1}$ and $E_{2}$ in $\Omega$, and we denote it by $d_{\Omega}\left(E_{1}, E_{2}\right)$.

A quadrilateral is a Jordan region $Q$ together with four points on its boundary. These points divide the boundary into two pairs of opposite sides $\alpha, \alpha^{\prime}$ and $\beta, \beta^{\prime}$. We can choose $\alpha, \alpha^{\prime}$ as the base pair and determine the extremal distance $d_{Q}\left(\alpha, \alpha^{\prime}\right)$.

Since the extremal distance is conformally invariant, we can replace $Q$ by a conformally equivalent rectangle $R$. We choose the mapping so that the $\alpha$, $\alpha^{\prime}$ side of $R$ lie on $x=0, x=a$ and the $\beta, \beta^{\prime}$ sides lie on $y=0, y=b$.

It is well known, see $[\mathrm{AH}]$, that $d_{Q}\left(\alpha, \alpha^{\prime}\right)=a / b$, and that $d_{Q}\left(\beta, \beta^{\prime}\right)=b / a$. Notice that $d_{Q}\left(\alpha, \alpha^{\prime}\right) \times d_{Q}\left(\beta, \beta^{\prime}\right)=1$.

There are other extremal lengths that can be associated with the configuration formed by $\Omega, E_{1}$ and $E_{2}$. We could let $\Gamma^{*}$ consist of all $\gamma^{*}$ in $\Omega$ which separate $E_{1}$ and $E_{2}$, the corresponding conformal invariant $\lambda_{\Omega}\left(\Gamma^{*}\right)$ is called the conjugate extremal distance of $E_{1}$ and $E_{2}$ with respect to $\Omega$, and is denoted by $d_{\Omega}^{*}\left(E_{1}, E_{2}\right)$.

In the quadrilateral case, we have the conjugate extremal distance equal to the reciprocal of the extremal distance. This is the case in all sufficiently regular cases.

It is well known that every doubly connected region is conformally equivalent to an annulus $R_{1}<|z|<R_{2}$. If $C_{1}$ and $C_{2}$ denote the two components of the boundary of an annulus $\Omega$, the extremal distance $d_{\Omega}\left(C_{1}, C_{2}\right)$ is equal to $(1 / 2 \pi) \ln \left(R_{2} / R_{1}\right)$. The conjugate extremal distance $d_{\Omega}^{*}\left(C_{1}, C_{2}\right)$ is equal to $2 \pi / \ln \left(R_{2} / R_{1}\right)$.

The importance of the extremal length in complex analysis derives not only from its conformal invariance, but also from the fact that it is comparatively easy to find lower and upper bounds for it. By definition, any specific choice of $\rho$ gives a lower bound for $\lambda_{\Omega}(\Gamma)$, namely,

$$
\lambda_{\Omega}(\Gamma) \geqq \frac{L(\Gamma, \rho)^{2}}{A(\Omega, \rho)} .
$$

We pass to list some of the properties of the extremal length that will be used throughout this paper. For a proof of these properties we refer the reader to the book "Conformal Invariants" $[\mathrm{AH}]$ by Ahlfors.

Theorem 2.1 (The comparison law). If every $\gamma \in \Gamma$ contains $\gamma^{\prime} \in \Gamma^{\prime}$, then $\lambda(\Gamma) \geqq \lambda\left(\Gamma^{\prime}\right)$.

As an immediate consequence of the comparison law we obtain the following corollary.

COROLlaRy 2.1.1. The extremal distance $d_{\Omega}\left(E_{1}, E_{2}\right)$ decreases when $\Omega, E_{1}, E_{2}$ increase.

THEOREM 2.2 (The composition laws). Let $\Omega_{1}$ and $\Omega_{2}$ be disjoint open sets. 
Let $\Gamma_{1}$ and $\Gamma_{2}$ consist of rectifiable arcs in $\Omega_{1}$ and $\Omega_{2}$, respectively, and let $\Gamma$ be a third set of rectifiable arcs.

1. If every $\gamma \in \Gamma$ contains $\gamma_{1} \in \Gamma_{1}^{\prime}$ and $\gamma_{2} \in \Gamma_{2}$, then

$$
\lambda(\Gamma) \geqq \lambda\left(\Gamma_{1}\right)+\lambda\left(\Gamma_{2}\right) .
$$

2. If every $\gamma_{1} \in \Gamma_{1}$ and every $\gamma_{2} \in \Gamma_{2}$ contains $\gamma \in \Gamma$, then

$$
\frac{1}{\lambda(\Gamma)} \geqq \frac{1}{\lambda\left(\Gamma_{1}\right)}+\frac{1}{\lambda\left(\Gamma_{2}\right)} \text {. }
$$

Theorem 2.3 (An Integral Inequality). Let $\Omega$ be a region in the complex plane between the lines $x=a$ and $x=b$. Denote by $\theta(t)$ the length of the intercept of $\Omega$ with the line $x=t$. Let $E_{1}$ and $E_{2}$ be those two boundary curves of $\Omega$ connecting the lines $x=a$ and $x=b$. Then,

$$
M_{\Omega}\left(E_{1}, E_{2}\right)=\frac{1}{d_{\Omega}\left(E_{1}, E_{2}\right)} \geqq \int_{a}^{b} \frac{d x}{\theta(x)} .
$$

We pass to describe some related results.

DEFINITION 2.1. Let $\Omega$ be a simply connected domain and $a, b$ a pair of points such that $b \in \Omega$ and $a \in \bar{\Omega}$. We define the reduced extremal length as follows.

For $\varepsilon>0$ let $\Gamma_{\varepsilon}$ denote the family of all curves joining the $\varepsilon$-neighborhoods of $a$ and $b$ in $\Omega$, and $\tilde{\Gamma}_{\varepsilon}$ the corresponding family in $\bar{C}$. Define,

$$
\beta=\beta(\Omega ; a, b)=\lim _{\varepsilon \rightarrow 0} \exp \left\{2 \pi\left[\lambda\left(\Gamma_{\varepsilon}\right)-\lambda\left(\tilde{\Gamma}_{\varepsilon}\right)\right]\right\},
$$

where $\lambda$ denotes the extremal length. The existence of the limit is a standard property of $\lambda$.

Let us now fix one of the points, say $b$, and consider $m$ distinct points $a_{1}$, $\ldots, a_{m}$ on $\partial \Omega$. Denote by $\beta=\beta\left(\Omega ; a_{\jmath}, b\right)$. Carleson and Makarov in [CM] showed that Brennan's conjecture is equivalent to the statement

$$
\sum_{j=1}^{m} \beta_{j}^{2} \leqq 1
$$

for any $m$ and any configuration $\left(\Omega ;\left\{a_{j}\right\}, b\right)$.

It is important to remark here that the $\beta$ 's are Möbius invariant, and they can be expressed in terms of conformal mappings.

Suppose that $b=\infty$ and let $f$, be a conformal map from $\boldsymbol{C} \backslash\{\boldsymbol{x}: \boldsymbol{x} \leqq \boldsymbol{0}\}$ onto $\Omega$ satisfying $f_{j}(\infty)=\infty$ and $f_{j}(0)=a_{\jmath}$. Suppose also that $f_{\jmath}$ has angular derivatives at 0 and $\infty$. Then,

$$
\beta_{\jmath}=\frac{\left|f_{j}^{\prime}(0)\right|}{\left|f_{j}^{\prime}(\infty)\right|} .
$$


Let again $b=\infty$ and let $f$ map the upper half plane onto $\Omega$ satisfying

$$
f(z) \approx z^{2}
$$

as $z \rightarrow \infty$. Suppose the points $x_{j} \in \boldsymbol{R}$ are such that

$$
f\left(x_{j}\right)=a_{\jmath}, \quad f^{\prime}\left(x_{\jmath}\right)=0,
$$

and the derivative $f^{\prime \prime}\left(x_{\jmath}\right)$ exists. Then

$$
\beta_{j}=\frac{2}{\left|f^{\prime \prime}\left(x_{\jmath}\right)\right|} \text {. }
$$

Indeed, if $\varepsilon$ is small, the preimage of $\left\{\left|w-a_{\jmath}\right|=\varepsilon\right\}$ is like a semicircle of radius $\sqrt{2 \varepsilon /\left|f^{\prime \prime}\left(x_{j}\right)\right|}$ and the preimage of $\{|w|=1 / \varepsilon\}$ is like a semicircle of radius $\varepsilon^{-1 / 2}$. Therefore, we have

$$
\begin{gathered}
\lambda\left(\tilde{\Gamma}_{\varepsilon}\right) \approx \frac{1}{2 \pi} \log \frac{1}{\varepsilon^{2}}, \\
\lambda\left(\Gamma_{\varepsilon}\right) \approx \frac{1}{\pi} \log \frac{1}{\varepsilon} \sqrt{\frac{\left|f^{\prime \prime}\left(x_{j}\right)\right|}{2}}
\end{gathered}
$$

and

$$
\beta_{\jmath}=\lim _{\varepsilon \rightarrow 0} e^{2 \pi\left[\lambda\left(\tilde{\Gamma}_{\varepsilon}\right)-\lambda\left(\Gamma_{\varepsilon}\right)\right]}=\frac{2}{\left|f^{\prime \prime}\left(x_{\jmath}\right)\right|} .
$$

Let $\Omega_{0}$ be a simply connected domain such that $\infty \in \Omega_{0}$ and the boundary $\Gamma_{0}$ consists of a finite number of straight line segments. Let $b, a_{1}, \ldots, a_{m}$ be the extreme points of $\Gamma_{0}$, that is, the points at which $\Omega_{0}$ makes the full angle. We assume that $b=0,\{x: x<0\} \subset \Omega_{0}$, and the segment $L$ of $\Gamma_{0}$ with endpoint at $b$ lies on the real axis. Then for every sufficiently small $k>0$ we can construct a fractal set, a "dandelion", $\Gamma(k)$ as follows.

For $j=1, \ldots, m$ let $l$, denote the segment of length $k$ lying on $\Gamma_{0}$ and ending at $a_{j}$. Define the polygon $\Gamma_{1}=\Gamma_{1}(k)$ by replacing each $l_{3}$ by a rescaled copy of $\Gamma_{0}$ so that under rescaling the segment $L$ corresponds to $l_{j}$. The polygon $\Gamma_{1}$ has $m^{2}$ extreme points other than $b$. To obtain $\Gamma_{2}=\Gamma_{2}(k)$ we repeat the above procedure with the scale $k^{2}$. Proceeding with the construction we define polygons $\Gamma_{2}, \Gamma_{3}, \ldots$ which converge to some fractal set $\Gamma=\Gamma(k)$. Observe that if $k$ is small enough, then no intersections occur at any step of the construction. We will call the polygon $\Gamma_{0}$ the "initiator" of the fractal set $\Gamma$.

There is some relationship between the properties of the harmonic measure on $\Gamma$ and the $\beta$-numbers of the initiator. For a domain $\Omega$ and $\varepsilon>0$ let us denote

$$
\gamma_{\Omega}(\varepsilon)=\limsup _{\rho \rightarrow 0} \frac{\log N(\rho, \varepsilon)}{|\log \rho|}
$$

where $N(\rho, \varepsilon)$ is the maximal number of disjoint discs of radius $\rho$ and harmonic measure at least $\rho^{1 / 2+\varepsilon}$. By a theorem in $[\mathrm{CM}]$ we have that 


$$
\gamma_{\Omega}(\varepsilon) \leqq K \varepsilon .
$$

Then we have the following two results:

THEOREM 2.4. 1) Let $\left\{\Gamma_{k}\right\}_{k}>0$ be the family of dandelions with initiator $\Gamma_{0}$, $\Omega_{k}=\bar{C} \backslash \Gamma_{k}$, and $\left\{\beta_{j}\right\}$ be the $\beta$-numbers of $\Gamma_{0}$. Then if

$$
\sum_{j=1}^{m} \beta_{j}^{p} \geqq 1
$$

for some $p>0$, then

$$
K \geqq \liminf _{k \rightarrow 0} \sup _{\varepsilon>0} \varepsilon^{-1} \gamma_{\Omega_{k}}(\varepsilon) \geqq 2 p .
$$

2) In the opposite derection, let

$$
\gamma_{\Omega}(\varepsilon)>K \varepsilon
$$

for some simply connected domain $\Omega$ and $\varepsilon>0$. Then, there exists a polygon $\Gamma_{0}$ satisfying

$$
\sum_{j=1}^{m} \beta_{j}^{K / 2} \geqq 1
$$

COROLlary 2.4.1. There exists an absolute constant $p$ such that

$$
\sum_{j=1}^{m} \beta_{\jmath}^{p} \geqq 1
$$

for every configuration $\left(\Omega ; b,\left\{a_{j}\right\}\right)$.

\section{Upper bounds}

We are going to concentrate on the following symmetric "dandelions".

Let $a_{h}=e^{\imath(h \pi /(k+1))}, S_{h}$ to be the line segment joining 0 and $a_{h}$ for any $h=$ $0, \pm 1, \pm 2, \ldots, \pm k$ and $B=(-\infty, 0]$.

Let $S=\cup_{h=-k}^{k} S_{h}$ and $\Theta=C-(S \cup B)$. Then $\Theta$ is a dandelion-like domain with $(2 k+1)$ branches equally spaced in the unit disk, as shown below

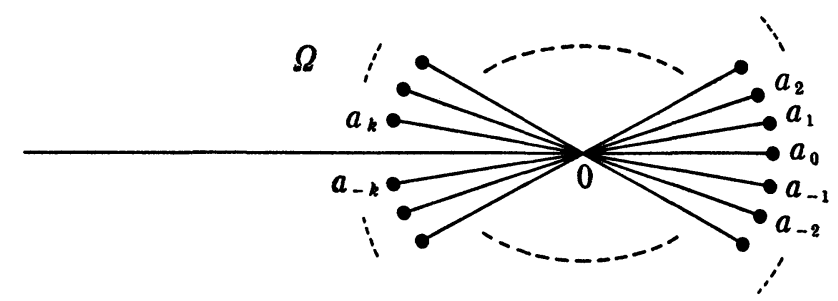

Let $H=C-B$. By the Riemann Mapping Theorem, for each $h=0, \pm 1, \ldots$, 
$\pm k$, there exists a conformal mapping $F_{h}(z): \Theta \rightarrow H$ with the following two normalizing conditions

$$
F_{h}\left(a_{h}\right)=0
$$

and

$$
\lim _{z \rightarrow \infty} \frac{F_{h}(z)}{z}=1
$$

Our goal in this section is to obtain estimates for the sum

$$
\sum_{h=-k}^{k} \beta_{h}
$$

where

$$
\beta_{h}=\left|F_{h}^{\prime}\left(a_{h}\right)\right|=\lim _{z \rightarrow a_{h}}\left|\frac{F_{h}(z)}{z-a_{h}}\right|, \quad h=0, \pm 1, \ldots, \pm k .
$$

Let $D_{\rho}^{h}=\left\{z:\left|z-a_{h}\right|<\rho\right\}$ and $D_{1 / \rho}^{h}=\left\{z:\left|z-a_{h}\right|<(1 / \rho)\right\}$. Let $\Theta_{\rho}^{h}=\left[\Theta \cap D_{1 / \rho}^{h}\right]$ $-\overline{D_{\rho}^{h}}$, where $\rho$ is a small positive number, and $h=0, \pm 1, \ldots, \pm k$ as before.

Let $H_{\rho}^{h}$ be the image of $\Theta_{\rho}^{h}$ under $F_{h}(z)$. By the normalizing conditions (3.1) and (3.2), we have that for any positive $\varepsilon$ there exists a positive $\rho(\varepsilon)$, call it $\rho$, such that

$$
\left\{z:|z|<\frac{1}{\rho}(1-\varepsilon)\right\} \cap H \subset F_{h}\left(D_{1 / \rho}^{h}\right) \subset\left\{z:|z|<\frac{1}{\rho}(1+\varepsilon)\right\} \cap H
$$

and

$$
\left\{z:|z|<\left|F_{h}^{\prime}\left(a_{h}\right)\right| \rho(1-\varepsilon)\right\} \cap H \subset F_{h}\left(D_{\rho}^{h}\right) \subset\left\{z:|z|<\left|F_{h}^{\prime}\left(a_{h}\right)\right| \rho(1+\varepsilon)\right\} \cap H
$$

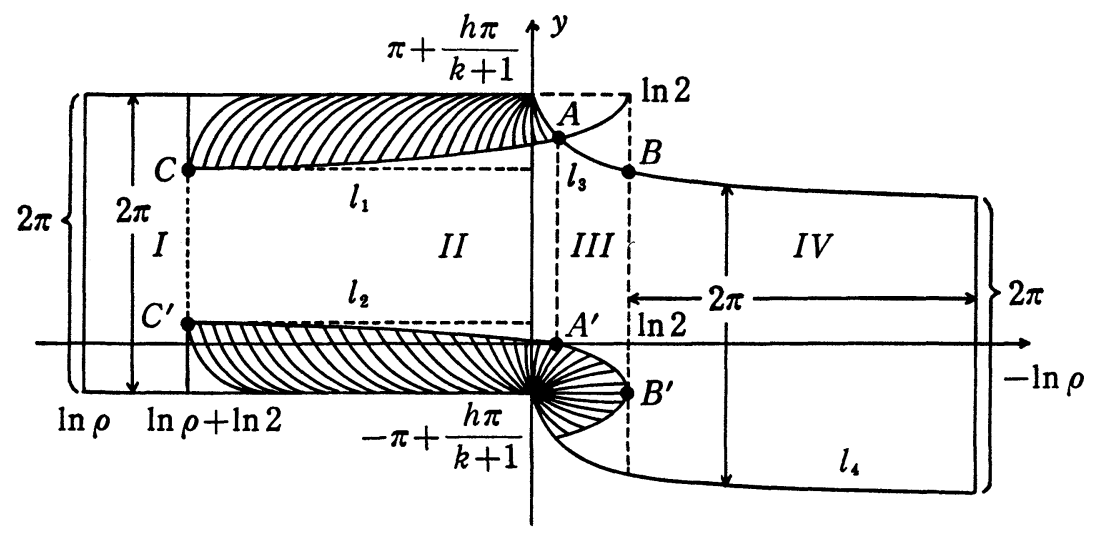

Since $F_{h}(z)$ is a conformal mapping from $\Theta \rightarrow H$, if we let $\Gamma$ be the family of curves joining $\partial D_{\rho}^{h}$ and $\partial D_{1 / \rho}^{h}$ in $\Theta_{\rho}^{h}$ by the conformal invariance of the 
modulus we have that $M\left(\Gamma, \Theta_{\rho}^{h}\right)=M\left(\Gamma^{\prime}, H_{\rho}^{h}\right)$, where $\Gamma^{\prime}$ is the family of curves joining $\partial F_{h}\left(D_{\rho}^{h}\right)$ and $\partial F_{h}\left(D_{1 / \rho}^{h}\right)$ in $H_{\rho}^{h}$.

Our next goal is to get an upper bound for $M\left(\Gamma, \Theta_{\rho}^{h}\right)$. To do this, we take the conformal mapping $\Phi_{h}(z)=\ln \left(z-a_{h}\right): \Theta_{\rho}^{h} \rightarrow S_{\rho}^{h}$, where $S_{\rho}^{h}=\Phi_{h}\left(\Theta_{\rho}^{h}\right)$ turns out to be a quadrangle as shown above.

First, notice that $\overline{F_{h}(\bar{z})}$ satisfies the two normalization conditions for $a_{-h}$, therefore $F_{-h}(z)=\overline{F_{h}(\bar{z})}$ and thus

$$
\sum_{h=-k}^{k} \beta_{h}=2 \sum_{h=1}^{k} \beta_{h}+\beta_{0} .
$$

Let $\bar{\Gamma}$ be the family of curves connecting the two vertical sides of the quadrangle $S_{\rho}^{h}$. By the conformal invariance of the modulus we have

$$
M\left(\Gamma, \Theta_{\rho}^{h}\right)=M\left(\bar{\Gamma}, S_{\rho}^{h}\right) .
$$

Let $\tilde{\Gamma}$ be the family of curves connecting the horizontal sides of $S_{\rho}^{h}$. By the conjugate rule we have

$$
M\left(\bar{\Gamma}, S_{\rho}^{h}\right)=\frac{1}{M\left(\tilde{\Gamma}, S_{\rho}^{h}\right)} .
$$

Therefore, to find a lower bound for $M\left(\Gamma, \Theta_{\rho}^{h}\right)$ we simply need to find a lower bound for $M\left(\tilde{\Gamma}, S_{\rho}^{h}\right)$. Since we have that $M\left(\Gamma, \Theta_{\rho}^{h}\right)=M\left(\Gamma^{\prime}, H_{\rho}^{h}\right)$ the comparison rule gives us that

$$
M\left(\Gamma^{\prime}, H_{\rho}^{h}\right) \geqq \frac{2 \pi}{\ln \left[(1+\varepsilon) /\left(\rho^{2}(1-\varepsilon)\left|F_{h}^{\prime}\left(a_{h}\right)\right|\right)\right]},
$$

which gives a lower for $M\left(\Gamma, \Theta_{\rho}^{h}\right)$.

To find an upper bound for $M\left(\Gamma, \Theta_{f}^{h}\right)=1 /\left(M\left(\tilde{\Gamma}, S_{\rho}^{h}\right)\right)$ we simply need to find a lower bound for $M\left(\tilde{\Gamma}, S_{\rho}^{h}\right)$ which we will obtain using the Integral Inequality from Section 2,

$$
M\left(\tilde{\Gamma}, S_{\rho}^{h}\right) \geqq \int \frac{1}{\theta(x)} d x
$$

\subsection{Data of configuration}

In the figure for $S_{\rho}^{h}$ we note several important points and lines. Their coordinate values and equations are listed below.

For the lines

$$
\begin{aligned}
& l_{1}: y=\pi+\frac{h \pi}{k+1}-\arccos \frac{e^{x}}{2} \\
& l_{2}: y=-\pi+\frac{h \pi}{k+1}+\arccos \frac{e^{x}}{2} \\
& l_{3}: y=\pi+\arcsin \frac{\sin (h \pi /(k+1))}{e^{x}}
\end{aligned}
$$




$$
l_{4}: y=-\pi+\arcsin \frac{\sin (h \pi /(k+1))}{e^{x}} \text {. }
$$

For the points

$$
\begin{aligned}
& A:\left(A_{x}, A_{y}\right)=\left(\ln 2+\ln \cos \frac{h \pi}{2(k+1)}, \pi+\frac{h \pi}{2(k+1)}\right) \\
& A^{\prime}:\left(A_{x}^{\prime}, A_{y}^{\prime}\right)=\left(\ln 2+\ln \cos \frac{h \pi}{2(k+1)},-\pi+\frac{3 h \pi}{2(k+1)}\right) \\
& B:\left(B_{x}, B_{y}\right)=\left(\ln 2, \arcsin \frac{\sin (h \pi /(k+1))}{2}+\pi\right) \\
& B^{\prime}:\left(B_{x}^{\prime}, B_{y}^{\prime}\right)=\left(\ln 2,-\pi+\frac{h \pi}{k+1}\right) \\
& C:\left(C_{x}, C_{y}\right)=\left(\ln \rho+\ln 2, \frac{\pi}{2}+\frac{h \pi}{k+1}\right) \\
& C^{\prime}:\left(C_{x}^{\prime}, C_{y}^{\prime}\right)=\left(\ln \rho+\ln 2,-\frac{\pi}{2}+\frac{h \pi}{k+1}\right) .
\end{aligned}
$$

It is clear that on the intervals $[\ln \rho, \ln \rho+\ln 2]$ and $[\ln 2,-\ln \rho], \theta(x)=2 \pi$. Let us split the interval $[\ln \rho,-\ln \rho]$ into four subintervals as follows

$$
\begin{aligned}
I: & {[\ln \rho, \ln \rho+\ln 2] } \\
I I: & {\left[\ln \rho+\ln 2, \ln 2+\ln \cos \frac{h \pi}{2(k+1)}\right] } \\
I I I: & {\left[\ln \cos \frac{h \pi}{2(k+1)}, \ln 2\right] } \\
I V: & {[\ln 2,-\ln \rho] . }
\end{aligned}
$$

On the intervals $I$ and $I V$ we have that

$$
\int_{I} \frac{1}{\theta(x)} d x=\frac{\ln 2}{2 \pi}
$$

and

$$
\int_{I V} \frac{1}{\theta(x)} d x=\frac{-\ln \rho-\ln 2}{2 \pi}
$$

Hence

$$
\int_{I} \frac{1}{\theta(x)} d x+\int_{I V} \frac{1}{\theta(x)} d x=\frac{-\ln \rho}{2 \pi} .
$$

Now we split the problem into two cases since $A_{x}$ will have different signs for different values of $h$.

CASE 1. For values of $h$ such that $0<(h \pi /(k+1)) \leqq 2 \pi / 3$. In this case $A_{x}$ 
$\geqq 0$ and we divide the interval $[\ln \rho+\ln 2, \ln 2]$ into three pieces:

$$
I:[\ln \rho+\ln 2,0], \quad I I:\left[0, A_{x}\right], \quad I I I:\left[A_{x}, B_{x}\right] .
$$

CASE 2. For values of $h$ such that $2 \pi / 3<h \pi /(k+1)<\pi$. In this case $A_{x}$ $<0$ and we divide the interval $[\ln \rho+\ln 2, \ln 2]$ into the following three pieces:

$$
I:\left[\ln \rho+\ln 2, A_{x}\right], \quad I I:\left[A_{x}, 0\right], \quad I I I:\left[0, B_{x}\right] .
$$

CASE 1. Values of $h$ for which $0 \leqq h \leqq 2 k / 3$.

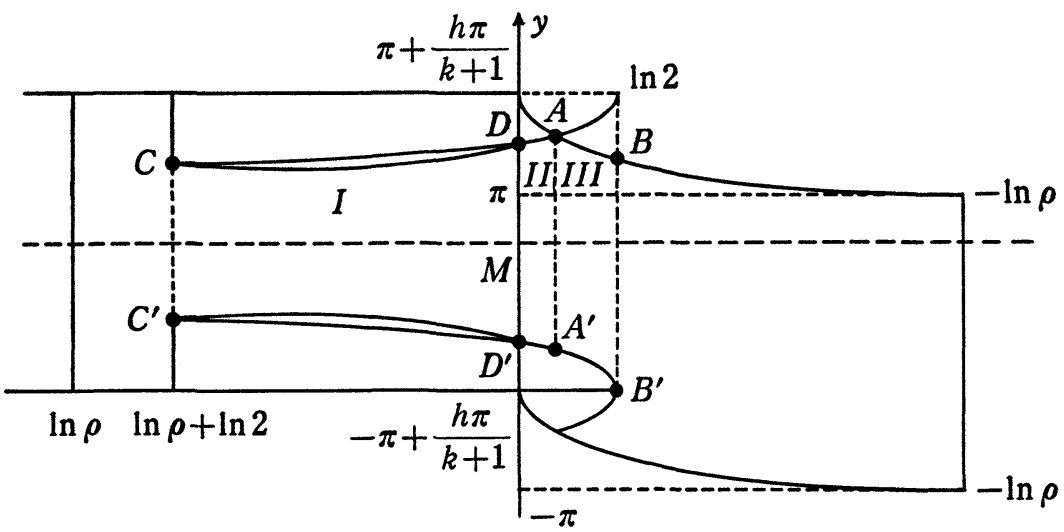

As shown in the figure above, we need the coordinate values for the points $D, D^{\prime}$, and $M$ along with the line equations for $C D, D A, A B$, and $A^{\prime} B^{\prime}$.

$$
D:\left(0, \frac{2 \pi}{3}+\frac{h \pi}{k+1}\right), \quad D^{\prime}:\left(0,-\frac{2 \pi}{3}+\frac{h \pi}{k+1}\right), \quad M:\left(0, \frac{h \pi}{k+1}\right) .
$$

We will use the slope intercept equation of a straight line $y=m x+b$. For the line $C D$ we have the following values for $m$ and $b$,

$$
\begin{gathered}
m=\frac{\pi / 6}{-\ln \rho-\ln 2} \\
b=\frac{2 \pi}{3}+\frac{h \pi}{k+1} .
\end{gathered}
$$

Thus,

$$
y=\frac{\pi / 6}{-\ln \rho-\ln 2} x+\frac{2 \pi}{3}+\frac{h \pi}{k+1} .
$$

For the line $D A$,

$$
m=\frac{(\pi / 3)-(h \pi / 2(k+1))}{\ln 2+\ln \cos (h \pi / 2(k+1))}
$$




$$
b=\frac{2 \pi}{3}+\frac{h \pi}{k+1} \text {. }
$$

Thus,

$$
y=\frac{(\pi / 3)-(h \pi / 2(k+1))}{\ln 2+\ln \cos (h \pi / 2(k+1))} x+\frac{2 \pi}{3}+\frac{h \pi}{k+1} .
$$

For the line $A B$,

$$
m=\frac{(h \pi / 2(k+1))-\arcsin (\sin (h \pi /(k+1)) / 2)}{\ln \cos (h \pi / 2(k+1))} .
$$

Thus,

$$
\begin{aligned}
y= & \frac{(h \pi / 2(k+1))-\arcsin (\sin (h \pi /(k+1)) / 2)}{\ln \cos (h \pi / 2(k+1))} x+\arcsin \frac{\sin (h \pi /(k+1))}{2} \\
& +\pi-\ln 2 \frac{(h \pi / 2(k+1))-\arcsin (\sin (h \pi /(k+1)) / 2)}{\ln \cos (h \pi / 2(k+1))} .
\end{aligned}
$$

For the line $A^{\prime} B^{\prime}$,

$$
m=\frac{h \pi / 2(k+1)}{\ln \cos (h \pi / 2(k+1))} .
$$

Thus,

$$
y=\frac{h \pi / 2(k+1)}{\ln \cos (h \pi / 2(k+1))} x+\frac{h \pi}{k+1}-\pi-\ln 2 \frac{h \pi / 2(k+1)}{\ln \cos (h \pi / 2(k+1))} .
$$

On the interval $I, \theta(x)$ is the distance between the lines $C D$ and $C^{\prime} D^{\prime}$, which is given by

$$
\begin{aligned}
\theta(x) & =2\left(\frac{\pi / 6}{-\ln \rho-\ln 2} x+\frac{2 \pi}{3}\right) \\
& =\frac{\pi / 3}{-\ln \rho-\ln 2} x+\frac{4 \pi}{3} .
\end{aligned}
$$

Thus

$$
\begin{aligned}
\int_{I} \frac{1}{\theta(x)} d x & =\int_{I} \frac{1}{((\pi / 3) /(-\ln \rho-\ln 2)) x+(4 \pi / 3)} d x \\
& =\frac{-\ln \rho-\ln 2}{\pi / 3}\left[\ln \left(\frac{\pi / 3}{-\ln \rho-\ln 2} x+\frac{4 \pi}{3}\right)\right]_{\ln \rho+\ln 2}^{0} \\
& =\frac{-\ln \rho-\ln 2}{\pi / 3}\left(\ln \frac{4 \pi}{3}-\ln \pi\right) \\
& =\frac{-\ln \rho-\ln 2}{\pi / 3} \ln \frac{4}{3} .
\end{aligned}
$$

On the interval $I I, \theta(x)$ is the distance between the lines $D A$ and $D^{\prime} A^{\prime}$, which is given by 
184

$$
\begin{aligned}
\theta(x) & =2\left(\frac{(\pi / 3)-(h \pi / 2(k+1))}{\ln 2+\ln \cos (h \pi / 2(k+1))} x+\frac{2 \pi}{3}\right) \\
& =\frac{(2 \pi / 3)-(h \pi /(k+1))}{\ln 2+\ln \cos (h \pi / 2(k+1))} x+\frac{4 \pi}{3} .
\end{aligned}
$$

Hence

$$
\begin{aligned}
\int_{I I} \frac{1}{\theta(x)} d x & =\int_{I I} \frac{1}{((2 \pi / 3)-(h \pi /(k+1)) /(\ln 2+\ln \cos (h \pi / 2(k+1)))) x+(4 \pi / 3)} d x \\
& =\frac{\ln 2+\ln \cos (h \pi / 2(k+1))}{(2 \pi / 3)-(h \pi /(k+1))} \\
& \times\left[\ln \left(\frac{(2 \pi / 3)-(h \pi /(k+1))}{\ln 2+\ln \cos (h \pi / 2(k+1))} x+\frac{4 \pi}{3}\right)\right]_{0}^{\ln 2+\ln \cos (h \pi / 2(k+1))} \\
& =\frac{\ln 2+\ln \cos (h \pi / 2(k+1))}{(2 \pi / 3)-(h \pi /(k+1))} \ln \left(\frac{2 \pi-(h \pi /(k+1))}{4 \pi / 3}\right) \\
& =\frac{\ln 2+\ln \cos (h \pi / 2(k+1))}{(2 \pi / 3)-(h \pi /(k+1))} \ln \left(\frac{2-(h /(k+1))}{4 / 3}\right) \\
& \geqq \frac{\ln 2}{2 \pi / 3} \ln \frac{3}{2} .
\end{aligned}
$$

On the interval $I I I, \theta(x)$ is the distance between the lines $A B$ and $A^{\prime} B^{\prime}$, which is given by

$$
\begin{aligned}
\theta(x)= & \frac{-\arcsin (\sin (h \pi /(k+1)) / 2)}{\ln \cos (h \pi / 2(k+1))} x+2 \pi+\arcsin \frac{\sin (h \pi /(k+1))}{2} \\
& -\frac{h \pi}{k+1}+\frac{\ln 2 \arcsin (\sin (h \pi /(k+1)) / 2)}{\ln \cos (h \pi / 2(k+1))} .
\end{aligned}
$$

Thus

$$
\begin{aligned}
\int_{I I I} \frac{1}{\theta(x)} d x= & \int_{I I I}\left(\frac{-\arcsin (\sin (h \pi /(k+1)) / 2)}{\ln \cos (h \pi / 2(k+1))}+2 \pi+\arcsin \frac{\sin (h \pi /(k+1))}{2}-\frac{k \pi}{k+1}\right. \\
& \left.+\frac{\ln 2 \arcsin (\sin (h \pi /(k+1)) / 2)}{\ln \cos (h \pi / 2(k+1))}\right)^{-1} d x \\
= & \frac{\ln \cos (h \pi / 2(k+1))}{-\arcsin (\sin (h \pi /(k+1)) / 2)}\left[\operatorname { l n } \left(\frac{-\arcsin (\sin (h \pi /(k+1)) / 2)}{\ln \cos (h \pi / 2(k+1))} x+2 \pi\right.\right. \\
& +\arcsin \frac{\sin (h \pi /(k+1))}{2}-\frac{h \pi}{k+1} \\
& \left.\left.+\frac{\ln 2 \arcsin (\sin (h \pi /(k+1)) / 2)}{\ln \cos (h \pi / 2(k+1))}\right)\right]_{\ln 2+\ln \ln \operatorname{los}(h \pi / 2(k+1))}^{\ln 2}
\end{aligned}
$$




$$
\begin{aligned}
& =\frac{\ln \cos (h \pi / 2(k+1))}{-\arcsin (\sin (h \pi /(k+1)) / 2)}\left[\ln \left(2 \pi+\arcsin \frac{\sin (h \pi /(k+1))}{2}-\frac{h \pi}{k+1}\right)\right. \\
& \left.-\ln \left(2 \pi-\frac{h \pi}{k+1}\right)\right] \\
& =\frac{\ln \cos (h \pi / 2(k+1))}{-\arcsin (\sin (h \pi /(k+1)) / 2)} \ln \left(1+\frac{\arcsin (\sin (h \pi /(k+1)) / 2)}{2 \pi-(h \pi /(k+1))}\right) \\
& \geqq \frac{\ln \cos (h \pi / 2(k+1))}{(h \pi /(k+1))-2 \pi} 4 \ln \frac{5}{4} \\
& \geqq \frac{\ln \cos (h \pi / 2(k+1))}{-2 \pi} 4 \ln \frac{5}{4} .
\end{aligned}
$$

Thus, when $0 \leqq h \leqq(2 / 3) k$ we have that

$$
\int_{C_{x}}^{B_{x}} \frac{1}{\theta(x)} d x \geqq \frac{-\ln \rho-\ln 2}{\pi / 3} \ln \frac{4}{3}+\frac{\ln 2}{2 \pi / 3} \ln \frac{3}{2}+\frac{\ln \cos (h \pi / 2(k+1))}{-2 \pi} 4 \ln \frac{5}{4} .
$$

CASE 2. Values of $h$ for which $(2 / 3) k \leqq h \leqq k$. In this case we can use a similar argument to get estimates for our integrals.

Now $A_{x}<0$, and as we mentioned before, we can divide the interval $[\ln \rho+$ $\ln 2, \ln 2]$ into three pieces,

$$
I:\left[\ln \rho+\ln 2, A_{x}\right] \quad I I:\left[A_{x}, 0\right] \quad I I I:\left[0, B_{x}\right] .
$$

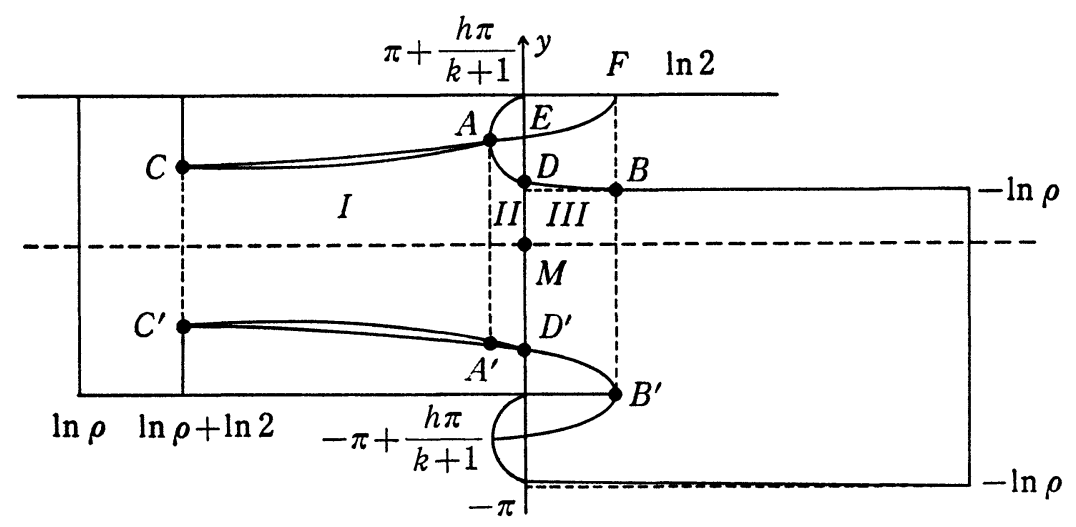

For intervals $I$ and $I I$ we can use the lines $C E$ and $C^{\prime} D^{\prime}$ to estimate $\theta(x)$ and from our previous calculation we have

$$
\int_{\mathrm{I}}+\int_{\Pi} \frac{1}{\theta(x)} d x=\frac{-\ln \rho-\ln 2}{\pi / 3} \ln \frac{4}{3} .
$$

For interval $I I I$ we use the lines $E F$ and $D^{\prime} B^{\prime}$ to estimate $\theta(x)$.

The coordinates of the points $E, F$, and $M$ are 


$$
E:\left(0, \frac{2 \pi}{3}+\frac{h \pi}{k+1}\right), \quad F:\left(\ln 2, \pi+\frac{h \pi}{k+1}\right), \quad M:\left(0, \frac{h \pi}{k+1}\right)
$$

and the equation of the line $E F$ is

$$
y=\frac{\pi / 3}{\ln 2} x+\frac{2 \pi}{3}+\frac{h \pi}{k+1},
$$

hence

$$
\begin{aligned}
\int_{I I I} \frac{1}{\theta(x)} d x & =\int_{0}^{\ln 2} \frac{1}{((\pi / 3) / \ln 2) x+2 \pi / 3} d x \\
& =\frac{3 \ln 2}{2 \pi}\left[\ln \left(\frac{2 \pi}{3 \ln 2} x+\frac{2 \pi}{3}\right)\right]_{0}^{\ln 2} \\
& =\frac{3 \ln 2}{2 \pi} \ln 2 .
\end{aligned}
$$

Therefore, when $(2 / 3) k \leqq h \leqq k$ we have that

$$
\int_{C_{x}}^{B_{x}} \frac{1}{\theta(x)} d x \geqq \frac{-\ln \rho-\ln 2}{\pi / 3} \ln \frac{4}{3}+\frac{3 \ln 2}{2 \pi} \ln 2 .
$$

Putting all these results together, we have:

CASE 1. Values of $h$ for which $0 \leqq h \leqq(2 / 3) k$.

$$
\begin{aligned}
\int_{\ln \rho}^{-\ln \rho} \frac{1}{\theta(x)} d x \geqq & \frac{-\ln \rho-\ln 2}{\pi / 3} \ln \frac{4}{3}+\frac{\ln 2}{2 \pi / 3} \ln \frac{3}{2} \\
& +\frac{\ln \cos (h \pi / 2(k+1))}{-2 \pi} 4 \ln \frac{5}{4}+\frac{-\ln \rho}{2 \pi} .
\end{aligned}
$$

CASE 2. Values of $h$ for which $(2 / 3) k \leqq h \leqq k$.

$$
\int_{\ln \rho}^{-\ln \rho} \frac{1}{\theta(x)} d x \geqq \frac{-\ln \rho-\ln 2}{\pi / 3} \ln \frac{4}{3}+\frac{3 \ln 2}{2 \pi} \ln 2+\frac{-\ln \rho}{2 \pi} .
$$

These equations give us a lower bound for $M\left(\tilde{\Gamma}, S_{\rho}^{h}\right)$ and therefore, we have

$$
\frac{1}{\int_{\ln \rho}^{-\ln \rho} \frac{1}{\theta(x)} d x} \geqq \frac{2 \pi}{\ln \left(\frac{1+\varepsilon}{\rho^{2}(1-\varepsilon)\left|F_{h}^{\prime}\left(a_{h}\right)\right|}\right)}
$$

which can be simplified to be

$$
\beta_{h} \leqq e^{\left.-2 \pi \int \ln \rho(1 / \theta)(x)\right) d x-2 \ln \rho} .
$$

This gives us an upper bound for the $\beta_{h}$ 's.

In Case 1 , we have

$$
\begin{aligned}
\beta_{h} & \leqq e^{(\ln \rho+\ln 2) 6 \ln (4 / 3)-3 \ln 2 \ln (3 / 2)-\ln \rho} \\
& \leqq e^{(6 \ln (4 / 3)-1) \ln \rho+6 \ln (4 / 3) \ln 2-3 \ln (3 / 2) \ln 2}
\end{aligned}
$$




$$
\leqq \rho^{6 \ln (4 / 3)-1} 2^{6 \ln (4 / 3)-3 \ln (3 / 2)} .
$$

In Case 2, we have

Hence,

$$
\begin{aligned}
\beta_{h} & \leqq e^{(\ln \rho+\ln 2) 6 \ln (4 / 3)-3 \ln 2 \ln 2-\ln \rho} \\
& \leqq \rho^{6 \ln (4 / 3)-1} 2^{6 \ln (4 / 3)-3 \ln 2}
\end{aligned}
$$

$$
\begin{aligned}
\beta_{0} & \leqq \rho^{6 \ln (4 / 3)-1} 2^{6 \ln (4 / 3)-3 \ln (3 / 2)} \\
\sum_{h=1}^{k} \beta_{h} & \leqq\left(\frac{2}{3} 2^{6 \ln (4 / 3)-3 \ln (3 / 2)}+\frac{1}{3} 2^{6 \ln (4 / 3)-3 \ln 2}\right) \rho^{6 \ln (4 / 3)-1} k \\
& \leqq C\left(\frac{1}{k}\right)^{6 \ln (4 / 3)-1} k \\
& \leqq C k^{0.273908}
\end{aligned}
$$

for some universal constant $C$ independent of $k$. Thus,

$$
\sum_{h=-k}^{k} \beta_{h} \leqq C \cdot k^{0.273908} \leqq \sqrt{k}
$$

for $k$ large enough. Showing that for our symmetric configurations

$$
\varlimsup_{k \rightarrow \infty}\left[\frac{\sum_{h=-k}^{h=k} \beta_{h}}{\sqrt{k}}\right]<1 \text {. }
$$

Therefore, these configurations will not work in an approach aimed to disprove Brennan's conjecture.

\section{Concluding remarks}

In all the calculations made in Section 3, we have used configurations in which all of the branches are of the same length and they are evenly distributed in the unit disk. It is still unknown if this will give us the best results for the upper bound. However, generally speaking, we know that this kind of symmetric configurations usually give the extreme values. An interesting problem will be to prove that our configurations give the upper bound for the sums $\sum_{h=-k}^{k} \beta_{h}$ among all possible configurations, that is, configurations with $k$ branches having different lengths, and randomly distributed over the unit disk.

Another interesting fact we would like to point out is that, when calculating the upper bounds for our $\beta_{h}$ 's, if we choose the best fit in our configuration we have

$$
\begin{aligned}
& \int_{\ln \rho}^{-\ln \rho} \frac{1}{\theta(x)} d x=\int_{\ln \rho}^{\ln \rho+\ln 2} \frac{1}{2 \pi} d x+\int_{\ln \rho+\ln 2}^{\ln 2+\ln \cos (h \pi / 2(k+1))} \frac{1}{2 \pi-2 \cos \left(e^{x} / 2\right)} d x+\int_{\ln 2}^{-\ln \rho} \frac{1}{2 \pi} d x \\
& +\int_{\ln 2+\ln \cos (h \pi / 2(k+1))}^{\ln 2} \frac{1}{2 \pi-(h \pi /(k+1))+\arcsin \left(\sin (h \pi /(k+1)) / e^{x}\right)-\arccos \left(e^{x} / 2\right)} d x,
\end{aligned}
$$


which is, according to our numerical evidence, very close to $-3 \ln \rho / 2 \pi$. If this observation can be proved, then we will have

$$
\begin{aligned}
\beta_{h} & \leqq e^{-2 \pi \int(1 / \theta(x)) d x-2 \ln \rho} \\
& \leqq e^{-2 \pi(-3 \ln \rho) / 2 \pi-2 \ln \rho} \\
& \leqq e^{\ln \rho} \\
& \leqq \rho .
\end{aligned}
$$

This leads us to make the following conjecture.

CONJECTURE 4.1. For any configuration similar to the ones described in this paper, (i.e. the lengths and the angles of the branches may vary) the sum of the $\beta_{h}$ 's is bounded from above by some universal constant $C$, namely

$$
\sum_{h=-k}^{k} \beta_{h} \leqq C .
$$

\section{REFERENCES}

[AH] Ahlfors, L. V., Conformal Invariants, Topics in Geometric Function Theory, McGraw-Hill Series in Higher Mathematics, 1973.

[Be] BAERNSTEIN II, A., A counterexample concerning integrability of derivatives of conformal mappings, J. Anal. Math., 53 (1989), 253-269.

[Br] BRENNAN, J., The integrability of the derivative in conformal mapping, J. London Math. Soc. (2), 18 (1978), 261-272.

[CM] Carleson, L. And Makarov, N.G., Some results connected with Brennan's conjecture, preprint.

[JO] Jenkins, J. A. And Oikawa, K., On results of Ahlfors and Hayman, lllinois J. Math., 15 (1971), 664-671.

[Po] Pommerenke, $\mathrm{CH}_{\mathrm{H}}$, On the integral means of the derivative of a univalent function, J. London Math. Soc. (2), 32 (1985), 254-258.

[V] Villamor, E., On a theorem of Baernstein, Ark. Mat., 33 (1995), 183-197.

Department of Mathematics

FLoRida INTERNATIONAL UNIVERSITY

MIAMI, FLORIDA 33199

U.S.A. 